\title{
Особенности электронного парамагнитного резонанса примеси железа в кристаллах $\mathrm{HgSe}$
}

\author{
() А.И. Вейнгер ${ }^{1}$, И.В. Кочман ${ }^{1}$, В.И. Окулов ${ }^{2, \uparrow, ~ Т . А . ~ Г о в о р к о в а ~}{ }^{2}$, М.Д. Андрийчук ${ }^{3}$, Л.Д. Паранчич 3 \\ ${ }^{1}$ Физико-технический институт им. А.Ф. Иоффе Российской академии наук, \\ 194021 Санкт-Петербург, Россия \\ ${ }^{2}$ Институт физики металлов им. М.Н. Михеева Уральского отделения Российской академии наук, \\ 620137 Екатеринбург, Россия \\ 3 Черновицкий национальный университет, \\ 58012 Черновцы, Украина \\ E-mail: kochman@mail.ioffe.ru
}

(Получена 10 сентября 2018 г. Принята к печати 17 сентября 2018 г.)

Рассмотрены спектры электронного парамагнитного резонанса ионов переходного металла $\mathrm{Fe}$ в матрице $\mathrm{HgSe}$. Их вид является стандартным для иона $\mathrm{Fe}^{3+}$, т. е. содержит 5 линий тонкой структуры с расщеплением, соответствующим слабому кристаллическому полю. Спектр наблюдается при температурах $T \leq 50 \mathrm{~K}$. Линии в спектрах искажены и имеют дайсоновскую форму. Анализ температурных зависимостей амплитуд линий показывает, что с понижением температуры происходит переход от парамагнетизма к ферромагнетизму с температурой Кюри $T \approx 7 \mathrm{~K}$.

DOI: 10.21883/FTP.2019.03.47281.8980

\section{1. Введение}

Электронный парамагнитный резонанс (ЭПР) в настоящее время является одним из широко применяемых методов исследования твердого тела. Из анализа спектров резонансного поглощения получают богатую информацию о магнитных моментах как невзаимодействующих, так и взаимодействующих ионов. При этом используют различные подходы для описания резонансных спектров в различных твердых телах: в ионных кристаллах, полупроводниках, полимерах, жидкостях и т.д.

С этой точки зрения примеси переходных металлов в кристаллах $\mathrm{HgSe}$ представляют собой интересный случай, поскольку они являются одновременно электрически активными и магнитоактивными примесями [1]. Кроме того, в металлическом состоянии переходный металл Fе проявляет ферромагнитные свойства. В связи с этим интересно выяснить, сохраняются ли ферромагнитные свойства, когда Fе входит в полупроводниковую матрицу в виде примесных ионов.

Ионы железа входят в решетку $\mathrm{HgSe}$ как примесь замещения. Они замещают атомы $\mathrm{Hg}$ и являются донорной примесью. При этом два $4 s$-электрона из внешней оболочки иона связываются с соседними атомами $\mathrm{Se}$, а оставшиеся образуют резонансный донорный уровень $E_{c}+0.02$ эВ на фоне сплошного спектра зоны проводимости. Электрон с этого уровня переходит в зону проводимости полупроводника, т. е. становится свободным. На системе электронов в зоне проводимости возможно наблюдение ЭПР в слабом магнитном поле, соответствующем их малой эффективной массе [2,3]. Кроме того, в результате потери одного электрона возникают ионы $\mathrm{Fe}^{3+}$, которые являются парамагнитными и также проявляются в ЭПР, но в более сильных полях, соответствующих иону $\mathrm{Fe}^{3+}$ [4]. В работе [5] было показано, что спектр ЭПР, обусловленный ионами $\mathrm{Fe}^{3+}$, состоит из 5 линий, которые определяются переходами между расщепленными кристаллическим полем спиновыми уровнями с проекциями от $-5 / 2$ до $+5 / 2$. При этом $g=2.007$ и параметр тонкой структуры $a=460 \cdot 10^{-4} \mathrm{~cm}^{-1}$.

В настоящей работе мы представляем результаты анализа спектров ЭПР ионов $\mathrm{Fe}^{3+}$ с целью выявления особенностей, обусловленных взаимодействием этих ионов.

\section{2. Образцы, методика измерений и обработка результатов}

Измерения проводились на образцах $\mathrm{HgSe}$, легированных $\mathrm{Fe}$, изготовленных в Черновицком национальном университете (Украина). Концентрация железа варьировалась от $10^{18}$ до $10^{21} \mathrm{~cm}^{-3}$. Это соответствует изменению удельного сопротивления в диапазоне $(1-3) \cdot 10^{-5}$ Ом·см [6]. Электрофизические характеристики образцов $\mathrm{HgSe}: \mathrm{Fe}$ более подробно описаны в [7].

В образцах с таким низким сопротивлением микроволновое поле, которое поглощается при резонансе, не проникает на всю глубину образца. Его воздействие ограничивается глубиной скин-слоя $\delta$. Оценки глубины скин-слоя по стандартным формулам показывают, что в этом случае $\delta=(1.5-3) \cdot 10^{-3}$ мм, т.е. $\sim 1$ мкм. Таким образом, микроволновое поле целиком сосредоточено вблизи поверхности образца. Это приводит к искажению линий резонансного поглощения [8].

Такое искажение делает невозможным определение концентрации спинов стандартным способом: путем двойного интегрирования спектра производной линии поглощения [8]. Анализ формы линии показал, что интегрирование является достаточно точным, если ин- 
тегрировать и сравнивать только положительные части производных линий образца и эталона [9].

Для исследований мы использовали ЭПР-спектрометр E-112 фирмы „VARIAN“ с проточным гелиевым криостатом „Oxford Instruments ESR-910“, который способен поддерживать температуру образца в широком диапазоне температур $(T=2-300 \mathrm{~K})$. Измерения проводились по классической ЭПР-методике. Единственная сложность при измерениях состояла в том, что сопротивление образца $R$ следовало поддерживать существенно бо́льшим или существенно меньшим, чем приведенное сопротивление волновода $\rho$, поскольку при $R \approx \rho$ чувствительность спектрометра заметно снижается $[8,9]$. В качестве эталонного образца использовался сертифицированный образец фирмы „Varian“ (раствор каменноугольной смолы в $\mathrm{KCl}$ ).

\section{3. Результаты эксперимента}

\section{1. Спектры ЭПР взаимодействующих ионов $\mathrm{Fe}^{3+}$ в $\mathrm{HgSe}$}

Вид спектра ЭПР образца $\mathrm{HgSe}$ с концентрацией железа $N_{\mathrm{Fe}}=7 \cdot 10^{18} \mathrm{~cm}^{-3}$ представлен на рис. 1 при трех различных направлениях магнитного поля: Н $\|[001]$,

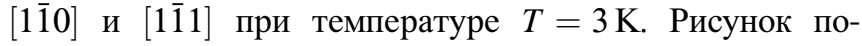
казывает, что спектр состоит из нескольких линий, пять из которых соответствуют классическому спектру ЭПР иона $\mathrm{Fe}^{3+}$. Этот спектр наблюдается на фоне производной магнитопоглощения, подробно описанного нами ранее [10]. Линии спектра были различимы на фоне шумов до $T=50 \mathrm{~K}$, однако при температурах $>20 \mathrm{~K}$ их обработка затруднялась из-за малой амплитуды.

Следует отметить, что магниторезистивный эффект всегда сопровождает ЭПР-поглощение при исследовании проводящих кристаллов. Особенно сильное изме-

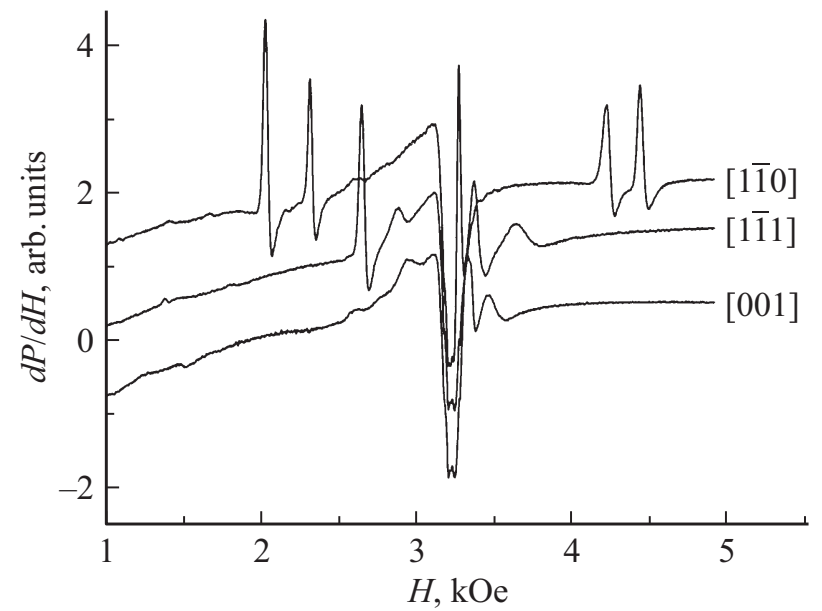

Рис. 1. Вид спектра ЭПР образца $\mathrm{HgSe}: \mathrm{Fe}$ с концентрацией $N_{\mathrm{Fe}}=7 \cdot 10^{18} \mathrm{~cm}^{-3}$ при $T=3 \mathrm{~K}$ и направлении магнитного поля вдоль трех главных кристаллографических осей: [110], [1111] и $[001]$.

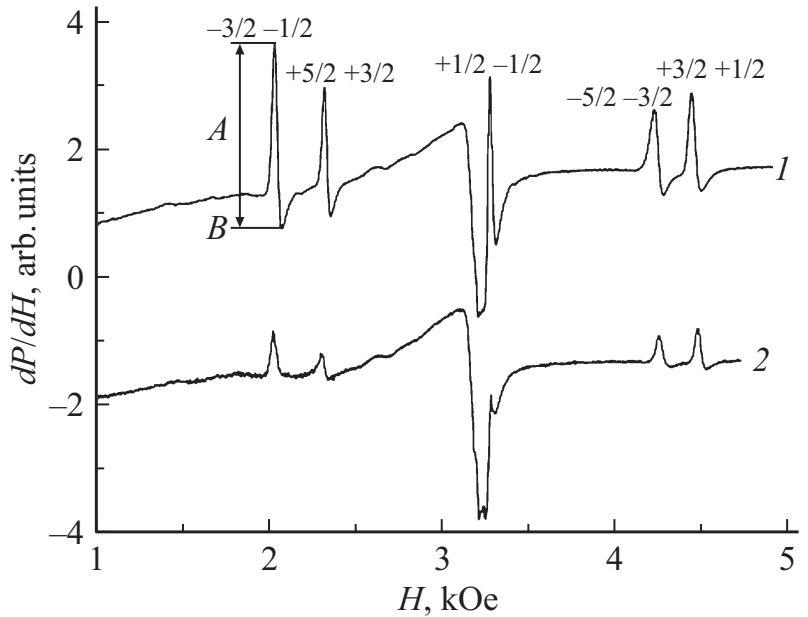

Рис. 2. Спектры ЭПР иона $\mathrm{Fe}^{3+}$ при направлении поля вдоль $[1 \overline{1} 0]$ и при концентрации спинов $N_{\mathrm{Fe}}=7 \cdot 10^{18}(1)$, $2 \cdot 10^{19} \mathrm{~cm}^{-3}(2) . T=3 \mathrm{~K}$.

нение сопротивления в магнитном поле наблюдается в полупроводниках с малой эффективной массой [11]. Кроме того, в спектре наблюдается фоновая линия значительной амплитуды, которая накладывается на центральную линию спектра $\mathrm{Fe}^{3+}$.

Описание спектра ЭПР $\mathrm{Fe}^{3+}$ основано на стандартном подходе, характерном для ионных кристаллов. В этом случае исходят из атомных уровней, а матрицу, в которую помещен парамагнитный атом, рассматривают как малое возмущение. Такой подход подробно рассмотрен в классических монографиях, из которых наиболее подходит для нашего случая монография [4]. В связи с этим для описания нашего спектра мы будем ей следовать. При таком подходе ион $\mathrm{Fe}^{3+}$ представляет собой ион переходного металла, на внешней оболочке которого находятся 5 вырожденных по спину электронов с параллельно направленными спинами. Слабое кристаллическое октаэдрическое поле снимает это вырождение, и в магнитном поле возможны пять переходов, обусловленных разностными энергиями между спиновыми уровнями с проекцией спина (квантовым числом) $M= \pm 5 / 2$, $\pm 3 / 2$ и $\pm 1 / 2$. В соответствии с этим в спектре $\mathrm{Fe}^{3+}$ наблюдаются 5 линий.

Они показаны на рис. 2 с обозначением соответствующих им резонансных переходов. Спектры такого вида наблюдались для двух концентраций $\mathrm{Fe}$ в $\mathrm{HgSe}$ : $N_{\mathrm{Fe}}=7 \cdot 10^{18}$ и $2 \cdot 10^{19} \mathrm{~cm}^{-3}$. В образце с концентрацией железа $\sim 10^{21} \mathrm{~cm}^{-3}$ сигнал ЭПР от ионов $\mathrm{Fe}^{3+}$ не наблюдался. Сравнение результатов показывает, что с ростом концентрации Fe амплитуда линий наблюдаемых спектров уменьшается, хотя соотношение между амплитудами линий в первом приближении сохраняется.

Магнитные поля для каждого резонансного перехода определяются соотношениями, представленными в таблице для направления $[1 \overline{1} 0]$, когда расстояние между линиями оказывается наибольшим. Через $a$ обозначена 
Резонансные переходы в $\mathrm{HgSe}$, легированном $\mathrm{Fe}$

\begin{tabular}{c|c|c}
\hline Переход & $\begin{array}{c}\text { Соотношение } \\
\text { для магнитного } \\
\text { поля }\end{array}$ & $\begin{array}{c}\text { Относительная } \\
\text { интенсивность } \\
\text { перехода }\end{array}$ \\
\hline$+5 / 2 \rightarrow+3 / 2$ & $h v=g \beta H+2 p a$ & 5 \\
$+3 / 2 \rightarrow+1 / 2$ & $h v=g \beta H-(5 / 2) p a$ & 8 \\
$+1 / 2 \rightarrow-1 / 2$ & $h v=g \beta H$ & 9 \\
$-1 / 2 \rightarrow-3 / 2$ & $h v=g \beta H+(5 / 2) p a$ & 8 \\
$-3 / 2 \rightarrow-5 / 2$ & $h v=g \beta H-2 p a$ & 5
\end{tabular}

энергия расщепления спиновых уровней кристаллическим полем. При повороте образца расстояние между линиями изменяется в зависимости от угла между направлениями магнитного поля и кристаллографической оси. Этот угол в таблице определяется параметром $p$.

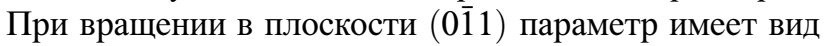

$$
p=1-5 \sin 2 \varphi+(15 / 4) \sin 4 \varphi,
$$

где угол $\varphi$ отсчитывается от направления [001].

Кроме того, в приведенных в таблице формулах мы пренебрегли членами 2-го порядка вида $(a / g \beta H)^{2}$ из-за их малости. Действительно, $a / g \beta=0.482$ кЭ и, следовательно, $(a / g \beta H)^{2} \sim 0.1$ или меньше. Полученное значение $a / g \beta$ хорошо согласуется с результатами, полученными в [5]. Из рис. 2 видно, что при увеличении концентрации до $N_{\mathrm{Fe}}=2 \cdot 10^{19} \mathrm{~cm}^{-3}$ значение $a / g \beta$ приблизительно сохраняется.

В таблице указаны и относительные интенсивности переходов из [4]. Качественно наши результаты согласуются с ними. Однако видно, что низкополевые линии гораздо интенсивнее высокополевых. Этот эффект может быть вызван несколькими причинами. Первая из них - это уширение резонансных линий в сильном поле. Действительно, если ширина самой низкополевой линии составляет 38 Э, то ширина самой высокополевой - 43 Э. В результате амплитуда высокополевых линий уменьшается, хотя второй интеграл от каждой из них должен иметь значение, более близкое к указанным в таблице.

Другая причина несоответствия теории и эксперимента определяется особенностями релаксации спинов, возбужденных внешним резонансным микроволновым полем. Это относительно длительный процесс. Поэтому происходит накопление спинов на верхнем уровне. В результате концентрация спинов на верхнем и нижнем уровнях сближается и разница между интенсивностями переходов, возбуждаемых полем с нижнего уровня на верхний и с верхнего уровня на нижний, уменьшается. Этот процесс и проявляется в уменьшении амплитуды линии поглощения. Особенно сильно этот эффект проявляется при прохождении высокополевых линий, которые обычно регистрируются после прохождения низкополевых линий.

К уменьшению разницы заселенности верхнего и нижнего спиновых уровней приводит и режим адиаба- тически быстрого прохождения, при котором верхний спиновый уровень оказывается более заселенным, чем нижний. При этом иногда экспериментально наблюдается переворот фазы производной линии поглощения, т. е. вместо резонансного поглощения наблюдается резонансное излучение. Возможно, что влияние этого процесса наблюдается при прохождении высокополевых линий, амплитуда которых оказывается заметно меньше амплитуды низкополевых линий. При прохождении низкополевых линий происходит увеличение заселенности верхних уровней и прохождение высокополевых линий происходит в условиях, когда температурное равновесие еще не восстановилось.

На рис. 2 виден известный для металлов эффект искажения производных линий спектра $\mathrm{Fe}^{3+}$. Вместо характерных для переходных ионов симметричных линий в нашем случае все линии, относящиеся к $\mathrm{Fe}^{3+}$, искажены. В них амплитуды низкополевых крыльев производных линий поглощения $\mathrm{Fe}^{3+}$ заметно больше амплитуд высокополевых крыльев. Такая форма линии называется дайсоновской $[11,12]$. Ее возможное происхождение мы обсудим далее.

\section{2. Температурная зависимость магнитной восприимчивости ионов $\mathrm{Fe}^{3+}$ как проявление их взаимодействия}

Хорошо известно (см., например, [13]), что температурные зависимости парамагнитной восприимчивости отражают процессы спинового взаимодействия. В диэлектриках, когда отсутствует взаимодействие спинов, температурные зависимости парамагнитной восприимчивости $\chi$ подчиняются закону Кюри

$$
\chi=C / T
$$

где $T$ - температура, $C$ - константа, определяющаяся концентрацией спинов.

При наличии взаимодействия в системе спинов парамагнетика температурные зависимости $\chi$ вблизи фазового перехода в ферромагнитное или антиферромагнитное состояние подчиняются закону Кюри-Вейсса

$$
\chi=C /(T-\Theta)
$$

где $\Theta$ - некоторая константа, имеющая размерность температуры, причем выше точки Кюри константа положительна, а выше точки Нееля отрицательна. Абсолютное значение $\Theta$ определяется температурами Кюри и Нееля соответственно.

Из спектра ЭПР можно найти магнитную восприимчивость, причем отдельно для каждого вида примесных центров, поскольку она определяется интегралом от линии резонансного поглощения:

$$
\chi \propto \int d H \int Y d H,
$$

где $Y$ - производная сигнала поглощения. 
Абсолютное значение магнитной восприимчивости из сигнала ЭПР обычно определяется путем сравнения с сигналом от эталонного образца.

В нашем случае найти точное значение магнитной восприимчивости оказалось невозможным по нескольким причинам. Во-первых, неизвестно точное значение сопротивления полупроводника, но оно мало, так что глубина скин-слоя существенно меньше толщины образца. Во-вторых, спектр ЭПР $\mathrm{Fe}^{3+}$ состоит из 5 линий, соотношение амплитуд которых считается приблизительно постоянным, но эксперимент показывает, что соотношение амплитуд линий меняется с температурой. В-третьих, на линии поглощения накладывается сигнал магнитосопротивления, который необходимо учитывать при интегрировании сигнала ЭПР. В-четвертых, как уже было отмечено выше, сами линии искажены из-за наличия скин-слоя. Наконец, в-пятых, следует учитывать изменение добротности резонатора с температурой, которое тоже влияет на амплитуду линий резонансного поглощения. В связи с этим при определении температурных зависимостей $\chi(T)$ были сделаны некоторые допущения, которые позволили упростить задачу.

Сравнение амплитуд резонансных линий тонкой структуры показало, что их соотношение остается приблизительно постоянным. Поэтому мы дважды интегрировали только одну линию, вызываемую переходом $-3 / 2 \rightarrow-5 / 2$ для образца с концентрацией $N_{\mathrm{Fe}}=7 \cdot 10^{18} \mathrm{~cm}^{-3}$, и считали, что остальные линии имели аналогичную температурную зависимость. Таким образом, вместо $\chi(T)$ мы рассматривали только ее часть, определяемую этой линией.

Эталонный образец находился в том же резонаторе, но вне криостата при комнатной температуре. Температурная зависимость второго интеграла от линии эталонного образца (рис. 3) показала, что он практически не зависит от температуры образца. Отсюда следует, что при изменении температуры добротность резонатора остается постоянной. Следовательно, сопротивление исследованного образца мало меняется с температурой и не влияет на величину сигнала.

Температурная зависимость магнитной восприимчивости изученного образца показана на рис. 4. Из него видно, что почти во всем диапазоне температур, где наблюдается сигнал ЭПР, зависимость $\chi(T)$ заметно отличается от закона Кюри. Это значит, что магнитные ионы $\mathrm{Fe}^{3+}$ взаимодействуют между собой.

При понижении температуры в области от 20 до $12 \mathrm{~K}$ наблюдается сверхлинейный рост магнитной восприимчивости, соответствующий закону Кюри-Вейсса с положительной константой $\Theta$. Действительно, если перестроить этот участок в виде $1 / \chi=f(T)$, то этот участок представляет собой прямую, пересекающую ось абсцисс в точке $T=\Theta \approx 7 \mathrm{~K}$. Этот участок в линейном масштабе показан на рис. 5.

Эта температура есть точка Кюри для данного образца, ниже которой кристалл становится ферромагнитным. В этом состоянии связь между внешним полем

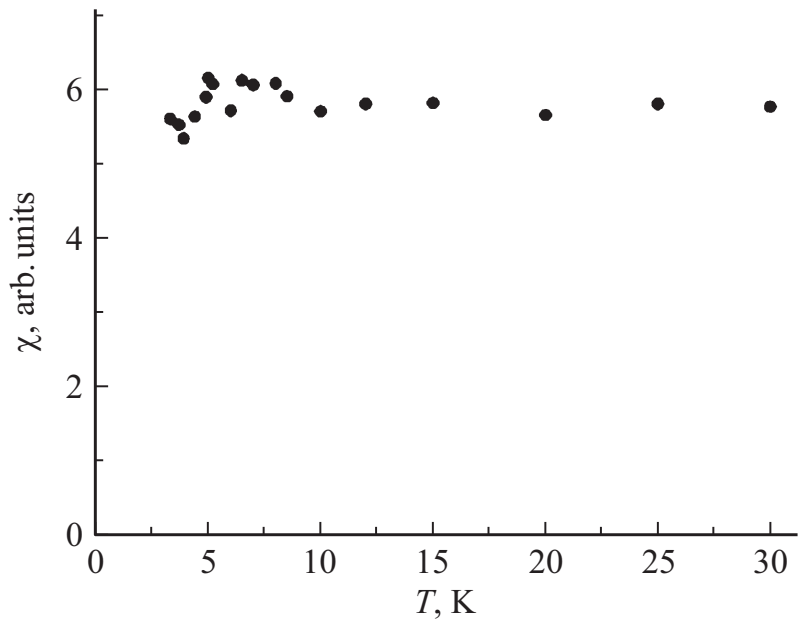

Рис. 3. Температурная зависимость магнитной восприимчивости эталонного образца.

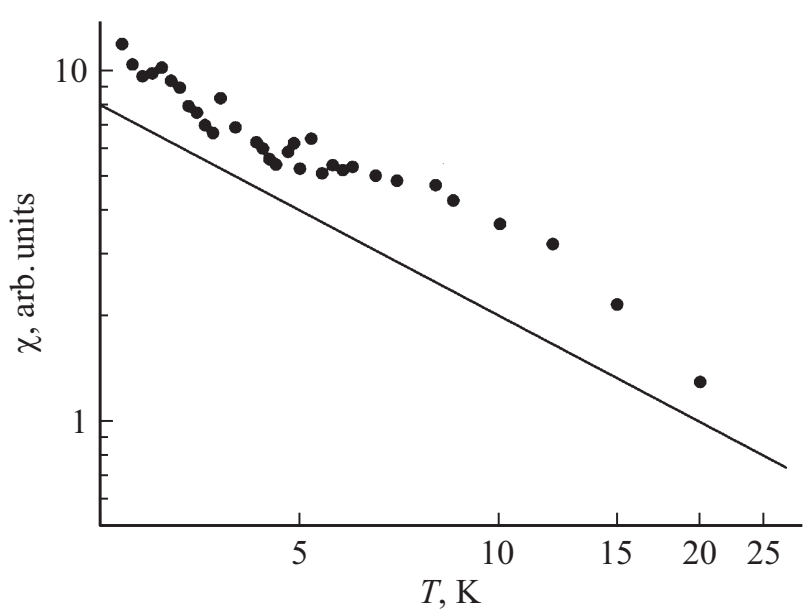

Рис. 4. Температурная зависимость магнитной восприимчивости, обусловленной примесью $\mathrm{Fe}^{3+}$ с концентрацией $N_{\mathrm{Fe}}=7 \cdot 10^{18} \mathrm{~cm}^{-3}$ в кристалле HgSe. Точки - эксперимент; линия соответствует температурной зависимости для закона Кюри.

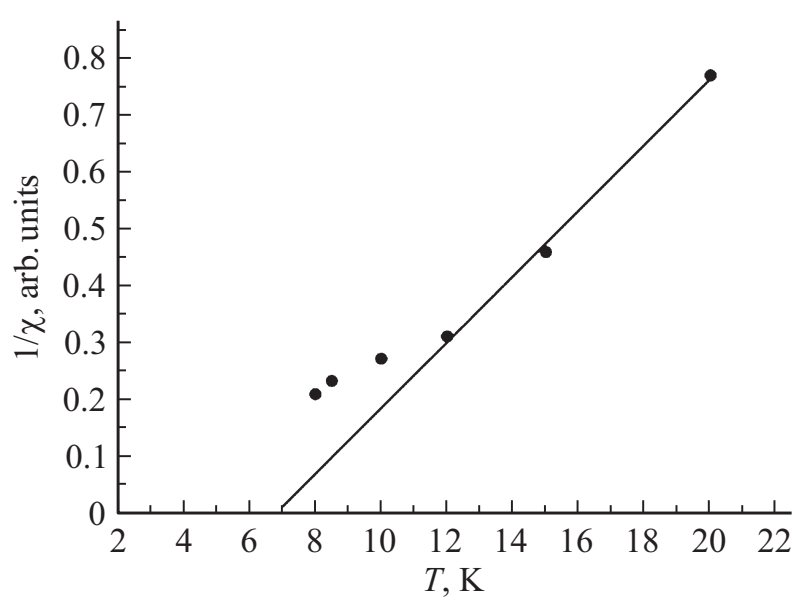

Рис. 5. Зависимость $1 / \chi=f(T)$ для исследованного образца. 
и намагниченностью образца становится нелинейной, и магнитная восприимчивость начинает зависеть не только от температуры, но и от внешнего поля.

К сожалению, методика ЭПР не позволяет находить кривую намагничивания, а только определяет связь между внешним полем и магнитным моментом в поле резонансного поглощения. Из рис. 4 видно, что ниже точки Кюри вплоть до температуры $T \approx 5 \mathrm{~K}$ магнитная восприимчивость практически не зависит от температуры, а при более низких температурах вновь начинается слабый рост намагниченности. Измерение намагниченности требует использования других методик и не является предметом исследования в настоящей статье.

Следует отметить, что, хотя эксперимент показывает наличие ферромагнитного взаимодействия при низких температурах, оно не приводит к появлению заметного дополнительного внутреннего магнитного поля, которое должно сдвигать спектры $\mathrm{Fe}^{3+}$ в слабые поля, как это происходит в классических ферромагнетиках.

Таким образом, температурная зависимость магнитной восприимчивости указывает на возникновение намагниченности в системе ионов $\mathrm{Fe}^{3+}$ при низких температурах. К сожалению, слабый сигнал ЭПР при больших концентрациях $\mathrm{Fe}^{3+}$ не позволил проследить за концентрационными зависимостями магнитной восприимчивости.

\section{3. Анализ дайсоновской формы линии ЭПР иона $\mathrm{Fe}^{3+}$}

Линия поглощения дайсоновской формы возникает при наличии ряда условий. Микроволновое поле не должно проникать на всю глубину образца, т.е. в образце должен существовать скин-слой малой глубины $\delta$, причем $\delta \ll D$, где $D-$ толщина образца. Спин преодолевает всю толщину образца за время $T_{D}$, а скин-слой за время $T_{\delta}$. Учитывается также время спин-решеточной релаксации $T_{2}$, которое определяет ширину линии поглощения.

Авторы работы [14], основываясь на теории Дайсона [12], построили номограммы зависимости отношения $T_{D} / T_{2}$ от отношения показанных на рис. 2 амплитуд низкополевого и высокополевого крыльев производной линии поглощения (см. также [8]). Для нашего случая и $T_{D} \gg T_{\delta}, T_{D} \gg T_{2}$ эта номограмма показана на рис. 6 .

Анализ рис. 2 дает отношение $A / B \approx 5.35$, откуда $\left(T_{\delta} / T_{2}\right)_{0.5} \approx 0.55$. Полуширина резонансной линии равна $\Delta H / 2=30$ Э, из которой определяется время $T_{2}=6 \cdot 10^{-9}$ с и время диффузии спина через скин-слой $T_{\delta}=2 \cdot 10^{-9}$ с. Этот результат означает, что спин $\mathrm{Fe}^{3+}$ не жестко локализован на своем ионе, а может время от времени перемещаться между соседними ионами, образуя примесную зону, подобно тому, как это происходит в $\mathrm{Ge}$ и $\mathrm{Si}$ вблизи фазового перехода изоляторметалл [15].

Медленная диффузия упорядоченных спинов является еще одним подтверждением появления спонтанной на-

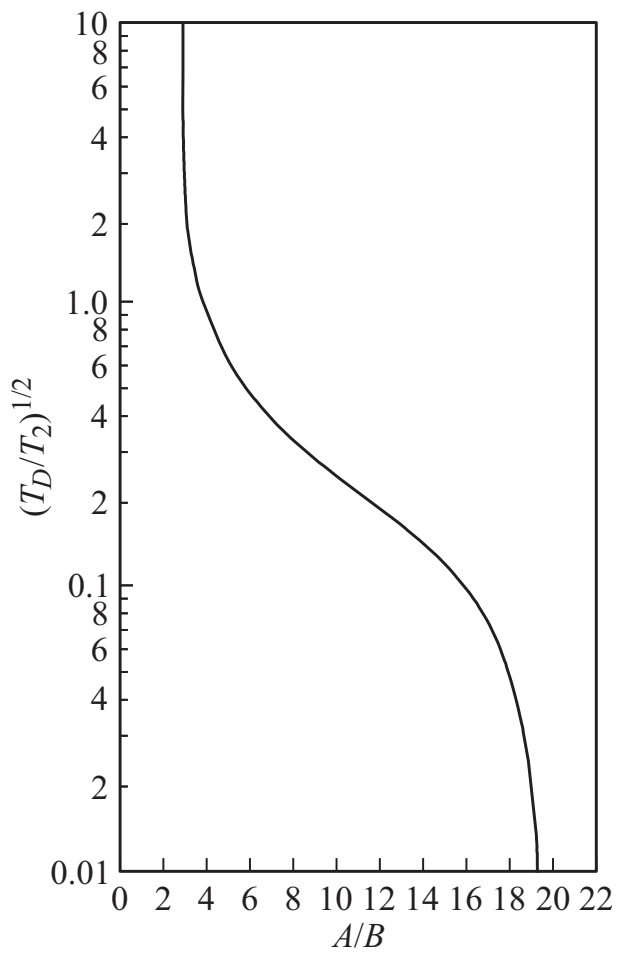

Рис. 6. Зависимость отношения времени диффузии через скин-слой к времени спин-решеточной релаксации от отношения низкополевого крыла $A$ к высокополевому крылу $B$.

магниченности в этом материале. Действительно, как показано [16], для появления спонтанной намагниченности необходимы два условия. Во-первых, спины магнитных ионов должны иметь параллельную ориентацию и, во-вторых, они должны быть тяжелыми, т.е. они должны иметь низкую подвижность. Этим условиям удовлетворяет совокупность примесных спинов $\mathrm{Fe}^{3+}$. Хорошо известно, как уже было отмечено выше, что каждый ион содержит пять электронов в $d$-оболочке, которые ориентированы параллельно. Связь между ионами при исследованной концентрации оказывается слабой, так что время спин-решеточной релаксации оказывается заметно больше, чем частота резонансного поглощения. Время диффузии через скин-слой также велико, так что электроны в этой зоне являются тяжелыми.

\section{4. Заключение}

Итак, спектр ЭПР ионов $\mathrm{Fe}^{3+}$ представляет собой набор из 5 линий тонкой структуры, что является типичным для этого иона в сильном магнитном поле, $H \gg a / g \beta$, где постоянная тонкой структуры $a=0.482$ кЭ.

Обнаружен эффект перехода линии Лоренца в линию Дайсона, которая ранее наблюдалась только для электронов в зоне проводимости. В нашем случае он возникает на тяжелых электронах в узкой примесной зоне ионов $\mathrm{Fe}^{3+}$. 
Определение температурной зависимости магнитной восприимчивости ионов $\mathrm{Fe}^{3+}$ показало, что она заметно отличается от закона Кюри. В некоторой области температур она подчиняется закону Кюри-Вейсса с положительной температурной константой $\Theta \approx 7 \mathrm{~K}$. Такую зависимость можно интерпретировать как результат возникновения намагниченности в системе ионов $\mathrm{Fe}^{3+} \mathrm{c}$ точкой Кюри $T_{\mathrm{C}}=7 \mathrm{~K}$.

Работа подготовлена при поддержке Программы президиума РАН „1.4. Актуальные проблемы физики низких температур“.

Авторы благодарны П.В. Семенихину и Д.А. Фролову за помощь в проведении экспериментов и участие в обсуждении.

\section{Список литературы}

[1] И.М. Цидильковский. УФН, 162, 63 (1992).

[2] Дж. Людвиг, Г. Вудбери. Электронный спиновый резонанс в полупроводниках (М., Наука, 1964).

[3] А.И. Вейнгер, И.В. Кочман, В.И. Окулов, М.Д. Андрийчук, Л.Д. Паранчич. ФТП, 52 (13), 1573 (2018).

[4] А. Абрагам, Б. Блини. Электронный парамагнитный резонанс переходных ионов (М., Мир, 1972) [Пер. с англ.: A. Abragam, B. Bleaney. Electron Paramagnetic Resonance of Transition Ions (Clarendon Press, Oxford, 1970)].

[5] Z. Wilamowski, A. Mycielski, W. Jantsch, G. Hendorfer. Phys. Rev. B, 38, 3621 (1988).

[6] A. Lenard, T. Dietl, M. Sawicki, W. Dobrowolski, K. Dybko, T. Dkoskiewicz, W. Plesiewicz, S. Miotkowska, A. Witek, A. Mycielski. J. Low Temp. Phys., 80, 15 (1990).

[7] А.Е. Лончаков, В.И. Окулов, С.Ю. Паранчич. ФНТ, 35, 92 (2009).

[8] Ч. Пул. Техника ЭПР спектроскопии (М., Мир, 1970) [Пер. с англ.: C.P. Poole. Electron spin resonance (Int. Pub. N.Y.-London-Sydney, 1967)].

[9] А.И. Вейнгер, А.Г. Забродский, Т.В. Тиснек, С.И. Голощапов, П.В. Семенихин. ЖТФ, 83 (12), 103 (2013).

[10] А.И. Вейнгер, И.В. Кочман, В.И. Окулов, М.Д. Андрийчук, Л.Д. Паранчич. ФТП, 52, 847 (2018).

[11] А.И. Вейнгер, А.Г. Забродский, Т.В. Тиснек, С.И. Голощапов. ФТП, 45, 1314 (2011).

[12] F.J. Dyson. Phys. Rev., 98, 349 (1955).

[13] Н. Ашкрофт, Н. Мермин. Физика твердого тела (М., Москва, 1979) [Пер. с англ.: N.V. Ashcroft, N.D. Mermin. Solid State Physics (Holt, Binenhart \& Winston N. Y., 1976)].

[14] G. Feher, A.F. Kip. Phys. Rev., 98, 337 (1955).

[15] Б.И. Шкловский, Ф.Л. Эфрос. Электронные свойства легированных полупроводников (М., Наука, 1979).

[16] С.В. Вонсовский. Магнетизм (М., Наука, 1971).

\section{Peculiarities of electron spin resonance of iron ions in $\mathrm{HgSe}$ crystals}

\author{
A.I. Veinger ${ }^{1}$, I.V. Kochman' ${ }^{1}$, V.I. Okulov', \\ T.A. Govorkova ${ }^{2}$, M.D. Andriichuk ${ }^{3}$, L.D. Paranchich ${ }^{3}$ \\ ${ }^{1}$ loffe Institute, \\ 194021 St. Petersburg, Russia \\ ${ }^{2}$ M.N. Miheev Institute of Metal Physics, \\ Ural Branch of Russian Academy of Sciences, \\ 620137 Ekaterinburg, Russia \\ ${ }^{3}$ Chernovtsy National University, \\ 48012 Chernovtsy, Ukraine
}

Abstract Electron spin resonance spectra of transition metal ions in the $\mathrm{HgSe}$ matrix are analyzed. The $\mathrm{Fe}^{3+}$ ion spectra consist of 5 fine structure components with a weak crystal field splitting observable at the temperatures up to $50 \mathrm{~K}$. The spectra lines are deformed and have a Dyson shape. An analysis of the temperature dependences of the lines amplitude shows that the transition from the paramagnetic ordering to the ferromagnetic ordering with decreasing temperature is occurred with the Curie temperature around $7 \mathrm{~K}$.

Редактор Л.В. Шаронова 